\title{
Diagnostic fibreoptic bronchoscopy in the immunocompromised host with pulmonary infiltrates
}

\author{
RICHARD A. MATTHAY, WAYNE C. FARMER, AND DIXON ODERO
}

From the Department of Medicine, Division of Pulmonary Medicine, Yale University School of Medicine, New Haven, Connecticut 06510, USA

\begin{abstract}
Matthay, R. A., Farmer, W. C., and Odero, D. (1977). Thorax, 32, 539-545. Diagnostic fibreoptic bronchoscopy in the immunocompromised host with pulmonary infiltrates. Nineteen immunocompromised patients with pulmonary infiltrates underwent diagnostic fibreoptic bronchoscopy with transbronchial forceps and brush biopsy. A specific diagnosis was obtained in $21 / 25$ procedures (10/11 focal lesions and 11/14 diffuse lesions). The most common diagnosis was infection, and organisms isolated included bacteria, fungi, Pneumocystis carinii, and herpes simplex. A pneumothorax requiring tube drainage occurred in two cases and mild lung parenchymal bleeding was noted in two others. It is concluded that fibreoptic bronchoscopy with forceps and brush biopsy can be performed safely with an excellent diagnostic yield in immunocompromised hosts with lung lesions. Supplemental oxygen should be administered during fibreoptic procedures in these patients and platelet transfusions should be given when thrombocytopenia is present.
\end{abstract}

Since its introduction in 1968, flexible fibreoptic bronchoscopy has been widely employed in the evaluation and management of patients with pulmonary disorders (Khan et al., 1976). The procedure is easily performed, is associated with few complications, and affords greater visualisation of the endobronchial tree than rigid bronchoscopy. Patients can undergo this procedure with minimal discomfort, and biopsy of previously inaccessible areas of the lung may be accomplished using the standard biopsy forceps accompanying the flexible fibreoptic bronchoscope.

Recently, several studies have demonstrated a high degree of diagnostic accuracy employing fibreoptic bronchoscopy for determining the aetiology of both focal and diffuse pulmonary infiltrates (Levin et al., 1974; Zavala, 1975; Cunningham et al., 1977). The safety and diagnostic yield of this procedure applied specifically to immunocompromised hosts with pulmonary lesions requires analysis. These patients are frequently at risk for lung biopsy procedures, particularly due to the presence of arterial hypoxaemia, abnormal clotting factors, and thrombocytopenia (Greenman et al., 1975; Hanson et al., 1976). The purpose of this study was to determine whether fibreoptic bronchoscopy employing brush and forceps biopsy is a safe and accurate method for determining the aetiology of pulmonary infiltrates in the immunocompromised patient.

\section{Material and methods}

Nineteen consecutive immunocompromised patients who underwent fibreoptic bronchoscopy for diagnosis of pulmonary infiltrates were studied prospectively. The study population was limited to patients who had established diagnoses of leukaemia, lymphoma, collagen vascular disease, organ transplantation or other diseases for which they had received corticosteroids, cytotoxic drugs, and/or radiation therapy. There were 13 men and six women with an average age of 44 , range 19 75 years. Underlying disease, characteristics of chest radiographs, arterial blood gas values, platelet count, prothrombin time, partial thromboplastin time, complications, diagnostic yield, and patient outcome were analysed.

Immunocompromised patients who developed pulmonary lesions were evaluated with cultures of sputum, transtracheal aspirates (Ries et al., 1974), blood, urine, and, if indicated, cerebrospinal fluid. If these failed to yield a diagnosis by appropriate stains and cultures fibreoptic bronchoscopy was 
performed. Procedures followed were those described by Levin et al. (1974) and Ellis (1975). Patients received nothing by mouth for at least six hours before the procedure. Premedications including codeine $(30-60 \mathrm{mg})$ and atropine $(0.4-0.6 \mathrm{mg})$ were given intramuscularly 30 minutes before the procedure. The patients' oropharynx and naris were anaesthetised with $4 \%$ lidocaine. In addition, viscous lidocaine was used to anaesthetise and lubricate the naris. The Olympus BF 5B2 fibreoptic bronchoscope was passed transnasally (Smiddy et al., 1971). Under direct vision, the vocal cords were examined and further anaesthetised with $2 \%$ lidocaine through the bronchoscope's suction and biopsy channel. The trachea was then entered and biopsy performed. When indicated, to ensure comfort and to facilitate the procedure diazepam (Valium), 5-10 mg, was administered intravenously. Topical anaesthetic (1\% lidocaine) was instilled into the airways with careful dose monitoring.

Supplemental oxygen was administered through a single-prong catheter attached to the naris (Petty et al., 1973). For one patient requiring assisted ventilation the bronchoscope was passed directly into the trachea through an endotracheal tube using an adaptor that provides a tight seal to maintain adequate tidal volume and ventilation (Amikam et al., 1972). Patients with platelet counts less than $50000 / \mathrm{mm}^{3}$ received platelet transfusions (10 platelet packs in most cases) during and immediately after the procedure to obtain platelet counts greater than $100000 / \mathrm{mm}^{3}$.

After complete examination of the tracheobronchial tree, including subsegmental bronchi, if no endotracheal lesions were seen, the bronchoscope was guided directly or by fluoroscopy into the segment containing the lesion for biopsy. Under fluoroscopic guidance, the standard biopsy forceps that accompanies the Olympus bronchoscope was passed peripherally in a closed position until mild resistance was met. The position of the forceps was carefully checked with fluoroscopy in both the lateral and anteroposterior projections. The proximity of the forceps to the visceral pleura was also noted. When satisfactory placement in the areas of involvement was achieved, the forceps was retracted 1 to $2 \mathrm{~cm}$ and opened, then advanced under fluoroscopic guidance into the lesion or within 1 to $2 \mathrm{~cm}$ of the visceral pleura in diffuse disease or until moderate resistance was encountered. The forceps was gently closed and slowly retracted while the bronchoscope was secured in the same position or slowly advanced to ensure accurate repositioning of the bronchial brush or forceps. The specimen was withdrawn through the bronchoscope's biopsy channel. Multiple biopsy specimens were taken from the same or different areas of involvement. Unilateral biopsies were done to prevent the possible occurrence of bilateral pneumothoraces. Bronchial brushings (Fennessey, 1966; Fry and ManaloEstrella, 1970; Repsher et al., 1972; Finley et al., 1974) of the same areas were performed and sent for cytological and microbiological examination. The biopsy specimens were placed in $10 \%$ formalin solution for histological examination. Additional specimens were placed in appropriate culture media.

Biopsy was performed in the radiology department using a single plane fluoroscopy unit with an image intensifying screen. Emergency equipment with cardiorespiratory arrest or pneumothorax management was available in all cases. Chest radiographs were taken just before the procedure and immediately afterwards.

\section{Results}

Nineteen patients underwent 25 fibreoptic bronchoscopy procedures. Underlying diseases are listed in Table 1. Risk factors for fibreoptic bronchoscopy are indicated in Table 2. Arterial oxygen tension among 16 hypoxaemia patients breathing ambient air ranged from 34 to 70 (average 55) $\mathrm{mmHg}$. Platelet counts in seven thrombocytopenic patients ranged from 2400 to $90000 / \mathrm{mm}^{3}$ (average $51000 / \mathrm{mm}^{3}$ ). Five patients with platelet counts less than $50000 / \mathrm{mm}^{3}$ received platelet transfusions during and immediately after bronchos-

Table 1 Immunocompromised patients (19) and underlying diseases

\begin{tabular}{ll}
\hline Underlying disease & No. of patients \\
\hline Hodgkin's disease & 6 \\
Other lymphoma & 2 \\
Acute myelogenous leukaemia & 4 \\
Other leukaemia & 1 \\
Renal transplant & 2 \\
Bronchogenic carcinoma & 1 \\
Multiple myeloma & 1 \\
Histiocytic medullary reticulosis & 1 \\
Systemic lupus erythematosus & 1
\end{tabular}

Table 2 Risk factors for fibreoptic bronchoscopy in immunocompromised hosts (25 procedures, 19 patients)

\begin{tabular}{lll}
\hline Risk factor & No. of procedures & No. of patients \\
\hline Arterial hypoxaemia & 18 & 16 \\
Thrombocytopenia & 9 & 7 \\
Abnormal partial & & \\
$\quad$ thromboplastin time & 4 & 4 \\
Abnormal prothrombin time & 3 & 3 \\
\hline
\end{tabular}


copy to obtain platelet counts greater than $100000 / \mathrm{mm}^{3}$. An abnormal prothrombin time, greater than three seconds beyond control, was noted in three patients, and an abnormal partial thromboplastin time, greater than 45 seconds, was identified in four.

\section{DIAGNOSTIC YIELD}

The results of fibreoptic bronchoscopy with brush and forceps biopsy are shown in Table 3. The diagnostic yield of brush biopsy was $63 \%$ and of transbronchial forceps biopsy $68 \%$. The combined diagnostic yield for both brush and forceps biopsies was $84 \%$ (21/25 procedures). Bronchoscopy results were confirmed subsequently by positive blood cultures, clearing of pulmonary infiltrates with appropriate therapy, open lung biopsy, or necropsy.

Table 4 indicates diagnoses obtained by fibreoptic bronchoscopy. Secondary neoplasms were all bronchogenic carcinomas. Two patients, aged 29 and 41 , had previously received radiation therapy to the thorax and intensive chemotherapy for Hodgkin's disease. The second tumours in these two patients developed in the previous radiation field. The third patient had received radiotherapy to the neck only for non-Hodgkin's lymphoma.

A specific infection was diagnosed by fibreoptic bronchoscopy in 14 patients. Table 5 shows that bacterial infection was identified most commonly followed by fungal organisms. A patient (RM) with histiocytic medullary reticulosis died with an invasive pulmonary Aspergillus fumigatus infection in spite of amphotericin B therapy (Fig. 1). A

Table 3 Diagnostic yield of fibreoptic bronchoscopy in immunocompromised hosts with pulmonary infiltrates (19 patients)

\begin{tabular}{llll}
\hline $\begin{array}{l}\text { Type of pulmonary } \\
\text { infiltripte }\end{array}$ & $\begin{array}{l}\text { Specific } \\
\text { diagnosis }\end{array}$ & $\begin{array}{l}\text { No. of } \\
\text { procedures }\end{array}$ & $\begin{array}{l}\text { Diagnostic } \\
\text { yield \% }\end{array}$ \\
\hline Diffuse & 11 & 14 & 79 \\
Focal & 10 & 11 & 91 \\
Total & 21 & 25 & 84 \\
\hline
\end{tabular}

Table 4 Diagnoses obtained by fibreoptic bronchoscopy in immunocompromised hosts with pulmonary infiltrates (25 procedures)

\begin{tabular}{lc}
\hline Diagnosis & No. of procedures \\
\hline Specific infection & 14 \\
Secondary neoplasm & 3 \\
Lung haemorrhage & 1 \\
Radiation pneumonitis & 1 \\
Lipoid pneumonitis & 1 \\
Mucous plug & 1 \\
No specific diagnosis & 4 \\
\hline
\end{tabular}

Table 5 Specific pulmonary infections (14) diagnosed in immunocompromised hosts and outcome

\begin{tabular}{lll}
\hline Infection & $\begin{array}{l}\text { No. of } \\
\text { procedures }\end{array}$ & $\begin{array}{l}\text { Infection } \\
\text { resolved }\end{array}$ \\
\hline Bacteria (Klebsiella 5, Nocardia 2, & & \\
$\quad$ Pseudomonas 1, Staphylococcus aureus 1) & 9 & $7 / 9$ \\
Fungus (Aspergillus fumigatus 1, & & $2 / 3$ \\
$\quad$ Aspergillus flavus 1, Candida albicans 1) & 3 & $0 / 1$ \\
Protozoan (Pneumocystis carinii) & 1 & $1 / 1$ \\
Virus (Herpes simplex) & 1 & \\
\hline
\end{tabular}

second patient (MM) with erythroleukaemia developed an invasive pulmonary $A$. flavus infection and recovered after amphotericin $B$ and rifampicin therapy (Fig. 2). Candida albicans was identified on transbronchial brush and forceps biopsy specimens from a patient with acute myelogenous leukaemia. The pulmonary nodular infiltrates cleared after treatment with amphotericin B. Pneumocystis carinii was isolated from brush and forceps biopsy specimens of a patient with stage IVB Hodgkin's disease after corticosteroids and cytotoxic chemotherapy had been discontinued. In spite of pentamidine therapy followed by pyramethamine and sulphadiazine therapy the patient died two months later with respiratory failure. At necropsy there was no evidence of Pneumocystis carinii in the lungs. Herpes simplex was identified on viral cultures of the lung in a patient receiving cytotoxic drugs for Hodgkin's disease (stage IVB), and the patient recovered spontaneously with clearing of the chest radiograph.

No specific diagnosis was obtained by fibreoptic bronchoscopy in four patients. Subsequently two of these patients died, and a necropsy revealed radiation pneumonitis in one. No necropsy was performed in the other patient. The pulmonary infiltrates cleared spontaneously in the third patient with Hodgkin's disease, and in the fourth case a diagnosis of pulmonary Nocardia infection was made on repeat fibreoptic bronchoscopy.

\section{COMPLICATIONS}

The morbidity of bronchoscopy with transbronchial forceps and brush biopsy was low. Pneumothorax requiring a chest tube occurred as a result of two procedures. Mild, transient haemoptysis with an increase in alveolar filling on the chest radiograph was noted in two other patients, and in neither instance was blood transfusion necessary. Both patients with bleeding had leukaemia and were thrombocytopenic with platelet counts of $2400 / \mathrm{mm}^{3}$ and $40000 / \mathrm{mm}^{3}$ respectively. In one the partial thromboplastin time was abnormal; however, in both, the pro- 


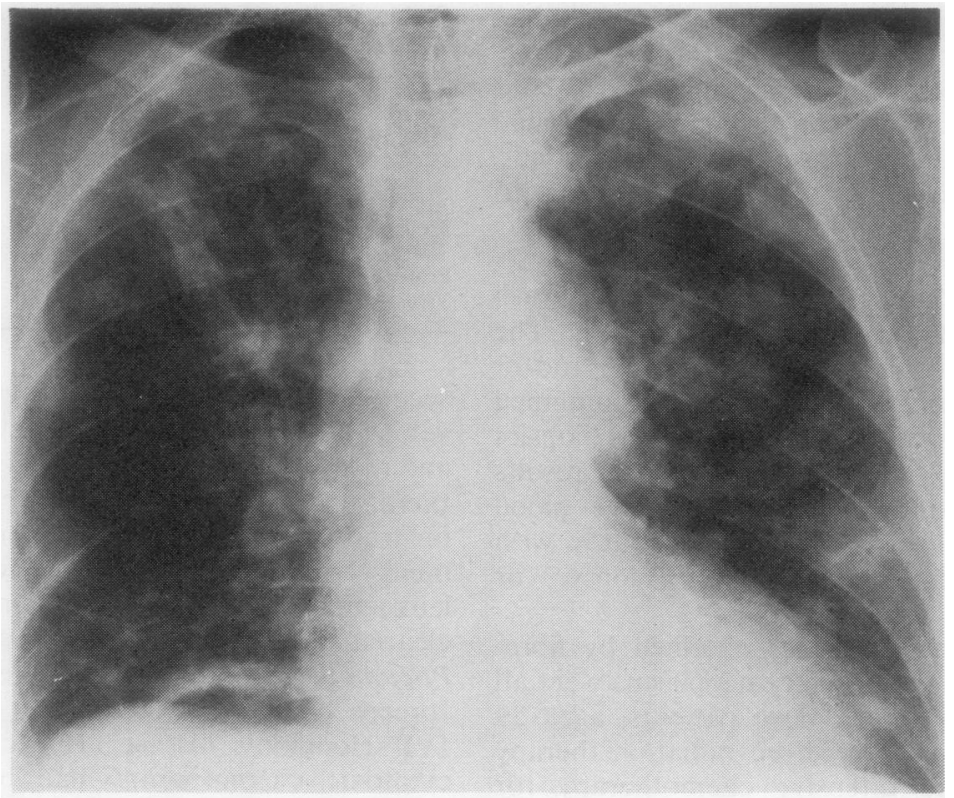

(a)

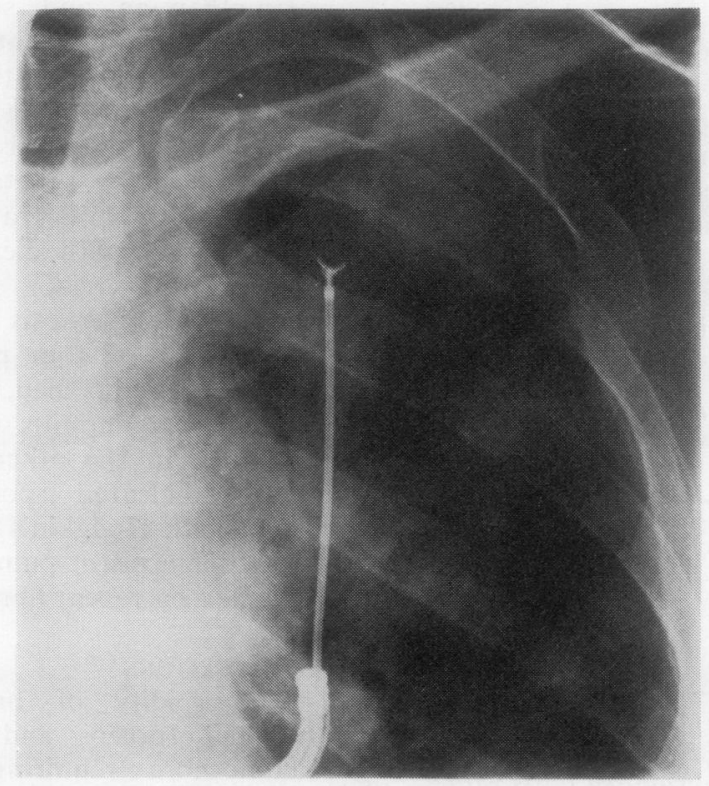

(b)

Fig. 1 Chest radiograph of a 65-year-old man (RM) with histiocytic medullary reticulosis receiving cytotoxic drugs and prednisone: (a) shows a bilateral, predominantly nodular infiltrate; (b) demonstrates the position of the biopsy forceps in the left upper lung zone during fibreoptic bronchoscopy. Aspergillus fumigatus was isolated on both transbronchial brush and forceps biopsies. Aspergillus precipitins rose from a titre of $1: 8$ to $1: 32$. 


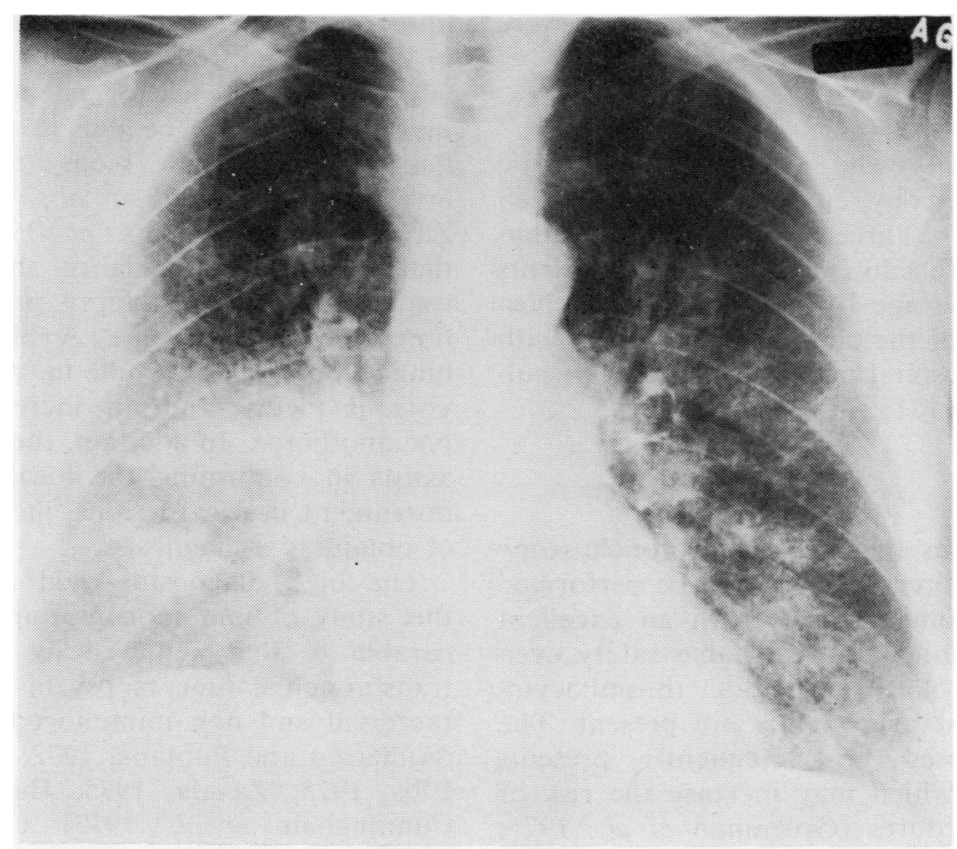

(a)

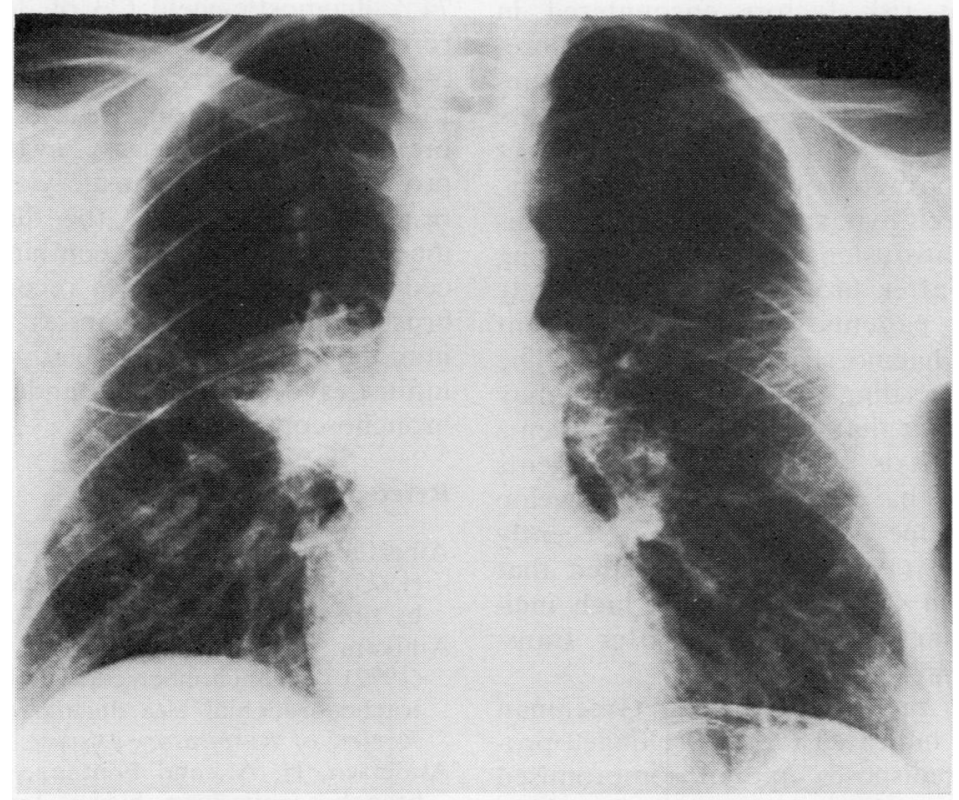

(b)

Fig. 2 Chest radiographs of a 52-year-old woman (MM) with erythroleukaemia receiving cytotoxic chemotherapy. Brush and transbronchial forceps lung biopsies revealed Aspergillus flavus, and A spergillus precipitins rose from a titre of 1:8 to 1:32: (a) shows diffuse, bilateral infiltrates; (b) after four weeks' therapy with amphotericin $B$ and rifampicin reveals considerable clearing of the pulmonary infiltrates. 
thrombin time was normal. No fatalities were attributed to fibreoptic bronchoscopy.

OUTCOME

Of the 19 patients who underwent diagnostic fibreoptic bronchoscopy, eight are still alive seven to 16 months later. Three deaths occurred within one week of the procedure and eight other patients died two to 20 (average 10) weeks after bronchoscopy. Resolution of the pulmonary infiltrates with discharge from hospital occurred in 10 of 14 pulmonary infections $(71 \%)$ (Table 5 ).

\section{Discussion}

This study confirms that fibreoptic bronchoscopy using brush and forceps biopsy can be performed in immunocompromised hosts with an excellent diagnostic yield and with acceptable safety even when abnormal clotting factors, thrombocytopenia, and arterial hypoxaemia are present. The immunocompromised host frequently presents special problems which may increase the risk of lung biopsy procedures (Greenman et al., 1975; Hanson et al., 1976; Cunningham et al., 1977). Arterial hypoxaemia and thrombocytopenia represent significant risk factors encountered in immunocompromised patients undergoing fibreoptic bronchoscopy (Hanson et al., 1976). Supplemental oxygen, found to be useful by others (Albertini et al., 1975; Zavala, 1975) to protect against severe hypoxaemia during bronchoscopy, appeared to protect hypoxaemic patients in this study. Platelet transfusions administered during and immediately after bronchoscopy in severely thrombocytopenic patients appeared to prevent severe pulmonary haemorrhage in our study. The low incidence of bleeding $(8 \%)$ in this series may be related to the fact that only two of 19 patients had renal failure. It is well known that patients with renal failure have a tendency to develop bleeding (Kazatchkine et al., 1976), and recently Cunningham et al. (1977) have emphasised that uraemic patients in their series had a high incidence of pulmonary haemorrhage after transbronchial biopsy $(45 \%)$.

In addition to thrombocytopenia, Greenman et al. (1975) have indicated that a prolonged prothrombin time predisposes immunocompromised hosts to pulmonary haemorrhage during lung biopsy procedures. In the current study only three patients had abnormal prothrombin times and none developed pulmonary haemorrhage. Our results suggest that with proper precautions, including platelet transfusions for thrombocytopenia and continuous supplemental oxygen, diagnostic fibreoptic bronchoscopy can be performed safely官 in immunocompromised patients.

The low incidence of pneumothorax observed흠 in our series may be due in part to the use of fluoroscopy to guide biopsy procedures. Several依 investigators (Levin et al., 1974; Ellis, 1975; Zavala, 1975; Hanson et al., 1976) have indicateds that fluoroscopic guidance should be used for? transbronchial lung biopsy procedures with the $\vec{\omega}$ fibreoptic bronchoscope. A blind transbronchialo lung biopsy may result in perforation of the visceral pleura and an increased incidence of pneumothorax. In addition, the use of fluoroscopy $\mathrm{N}$ assists in confirming the location of the biopsyor instrument in focal lesions, increasing the chancew of obtaining a diagnosis.

The high diagnostic yield obtained by us in this study of immunocompromised hosts is com-O parable to that achieved by others performing transbronchial lung biopsy in both immunocom-promised and non immunocompromised patients (Andersen and Fontana, 1972; Levin et al., 1974:0 Ellis, 1975; Zavala, 1975; Hanson et al., 1976; Cunningham et al., 1977). Cunningham et al. (1977) evaluated fibreoptic bronchoscopy specifi-క cally in immunosuppressed patients, obtaining a $74 \%$ diagnostic yield ( 23 of 31 procedures) with forceps transbronchial biopsy and a $28 \%$ yield (9 of 31 procedures) with bronchial brush biopsy. $\overrightarrow{\vec{A}}$ In the present study brush biopsy and trans-o bronchial forceps biopsy evaluated individuallys provided similar diagnostic yields of 63 and $68 \%$ respectively. However, the diagnostic yield was increased to $84 \%$ by combining the two pro-0 cedures. Therefore, we recommend that both brush and forceps biopsies be performed ino immunocompromised hosts with pulmonary. infiltrates who are undergoing fibreoptico bronchoscopy.

\section{References}

Albertini, R. E., Harrell, J. H., and Moser, K. M․ㅡㄹ (1975). Management of arterial hypoxemia induceof by fiberoptic bronchoscopy. Chest, 67, 134-136.

Amikam, B., Landa, J., West, J., and Sackner, M. A 응 (1972). Bronchofiberscopic observations of thew tracheobronchial tree during intubation. Americar Review of Respiratory Disease, 105, 747-755.

Andersen, H. A., and Fontana, R. S. (1972). Trans bronchoscopic lung biopsy for diffuse pulmonaryes diseases: technique and results in 450 cases. Chest 62, 125-128.

Cunningham, J. H., Zavala, D. C., Corry, R. J., andक्षे Keim, L. W. (1977). Trephine air drill, bronchia尽 brush and fiberoptic transbronchial lung biopsies in immunosuppressed patients. American Review oto Respiratory Disease, 115, 213-220. 
Ellis, J. H., Jr. (1975). Transbronchial lung biopsy via the fiberoptic bronchoscope: experience with 107 consecutive cases and comparison with bronchial brushing. Chest, 68, 524-532.

Fennessey, J. J. (1966). A technique for the selective catheterization of segmental bronchi using arterial catheters. American Journal of Roentgenology, 96, 936-943.

Finley, R., Kieff, E., Thomsen, S., Fennessy, J., Beem, M., Lerner, S., Morello, J. (1974). Bronchial brushing in the diagnosis of pulmonary disease in patients at risk for opportunistic infection. American Review of Respiratory Disease, 109, 379 387.

Fry, W. A., and Manalo-Estrella, P. (1970). Bronchial brushing. Surgery, Gynecology and Obstetrics, 130, 67-71.

Greenman, R. L., Goodall, P. T., and King, D. (1975). Lung biopsy in immunocompromised hosts. American Journal of Medicine, 59, 488-496.

Hanson, R. R., Zavala, D. C., Rhodes, M. L., Keim, L. W., and Smith, J. D. (1976). Transbronchial biopsy via flexible fiberoptic bronchoscope: Results in 164 patients. American Review of Respiratory Disease, 114, 67-72.

Kazatchkine, M., Sultan, Y., Caen, J. P., and Bariety, J. (1976). Bleeding in renal failure: A possible cause. British Medical Journal, 2, 612-615.

Khan, M. A., Whitcomb, M. E., and Snider, G. L. (1976). Flexible fiberoptic bronchoscopy. American Journal of Medicine, 61, 151-155.
Levin, D. C., Wicks, A. B., and Ellis, J. H., Jr. (1974). Transbronchial lung biopsy via the fiberoptic bronchoscope. American Review of Respiratory Disease, 110, 4-12.

Petty, T. L., Nett, L. M., and Lakshminarayan, S. (1973). A single nasal prong for continuous oxygen therapy. Respiratory Therapy, 18, 421.

Repsher, L. H., Schröter, G., and Hammond, W. S. (1972). Diagnosis of Pneumocystis carinii pneumonitis by means of endobronchial biopsy. New England Journal of Medicine, 287, 340-341.

Ries, K., Levison, M. E., and Kaye, D. (1974). Transtracheal aspiration in pulmonary infection. Archives of Internal Medicine, 133, 453-458.

Smiddy, J. F., Ruth, W. E., Kerby, G. R., Renz, L. E., and Raucher, C. (1971). Flexible fiberoptic bronchoscope (letter). Annals of Internal Medicine, 75, 971.

Zavala, D. C. (1975). Diagnostic fiberoptic bronchoscopy: techniques and results of biopsy in 600 patients. Chest, 68, 12-19.

Requests for reprints to: Dr. R. A. Matthay, Division of Pulmonary Medicine, Department of Medicine, Yale University School of Medicine, 333 Cedar Street, New Haven, Connecticut 06510, USA. 